\title{
Economic drug discovery and rational medicinal chemistry for tropical diseases*
}

\author{
Kelly Chibale $\ddagger$ \\ Department of Chemistry, University of Cape Town, Rondebosch 7701, South \\ Africa
}

\begin{abstract}
In order to fulfill research objectives around target-based drug discovery in the field of anti-infective agents that are prevalent mainly in poor Third World countries, selection of biological and chemical targets is guided by economic drug discovery and rational medicinal chemistry. Selection of biological targets of therapeutic relevance in multiple disease-causing organisms, as well as the use of natural products and existing drugs as chemical scaffolds for the discovery and design of novel therapeutics should be viable strategies underpinning drug discovery research in poor Third World countries. In this regard, biological targets of interest to our program include disulfide reductases and cysteine proteases (CPs), while chemical scaffolds include existing antimalarial agents and natural products.
\end{abstract}

Keywords: Malaria; trypanosomiasis; natural products; enzyme inhibitors; medicinal chemistry.

\section{INTRODUCTION}

Since the market for drugs against malaria, tuberculosis, AIDS, and trypanosomiasis is mainly in poor Third World countries, innovative and cost-effective approaches to drug discovery and development are urgently needed. Economic drug discovery entails, amongst other things, the development of single agents that provide inhibition of multiple disease-causing organisms in an analogous way to broadspectrum antibiotics. On the other hand, rational medicinal chemistry in this context at least involves the rational selection of a starting point for drug discovery.

Standard approaches to lead compound discovery include random and nonrandom (or targeted or focused) screening. In any case, natural products have long been recognized as excellent sources of potential lead compounds [1]. Nowadays, the development of a novel drug from natural sources might include: (a) screening of natural products for biological activity, (b) isolation and purification of the active principle(s), (c) determination of the chemical structure(s), (d) structure-activity relationship (SAR) studies, (e) synthesis of analogs, (f) mechanism of action studies, and (g) design and synthesis of novel drug structures. While natural products are indeed a good starting point for drug discovery, another useful starting point is an existing drug. As has been well stated by Sir James Black, "The most fruitful basis for the discovery of a new drug is to start with an old drug" [2]. To illustrate this important statement, an analysis of the origins of recently launched drugs revealed that most were derived by modification of the chemical structure of known drugs or lead structures from the literature [3].

\footnotetext{
*Paper based on a presentation at the $9^{\text {th }}$ International Chemistry Conference in Africa (ICCA-9), Arusha, Tanzania, 2-7 August 2004. Other presentations are published in this issue, pp. 1923-1964.

¥Fax: 2721689 7499: E-mail: chibale@ science.uct.ac.za
} 
In a meaningful drug discovery process, there should be guidelines that contribute to rational medicinal chemistry efforts including the consideration of practical issues of lead discovery and development. At this juncture, the difference between a hit molecule and a lead molecule should be clarified because most drug discovery projects in academic institutions do not consider even the most basic criteria for a lead molecule. By definition, a hit molecule is one that gives a positive result in a primary in vitro screen. On the other hand, a lead molecule is one that gives confidence that a preclinical candidate will be found. Some of the criteria that a lead compound should meet include demonstration of in vivo activity, SAR trends, known mechanism of action, a good pharmacokinetic profile, selectivity in related systems, and molecular simplicity, which may have a bearing on physical properties [4] and number of synthetic steps. The need for fewer synthetic steps cannot be overemphasized for tropical diseases.

\section{RESULTS AND DISCUSSION}

Our current medicinal chemistry program on novel anti-infective (antimalarial, antituberculosis, antiHIV/AIDS, and antitrypanosomal) and anticancer agents has four main objectives: (i) to develop targetdirected inhibitors, (ii) to develop single agents that provide target-directed inhibition of multiple disease-causing organisms or cells, (iii) to develop single agents that provide maximal anti-infective and anticancer activity by acting against multiple targets within the same cell, and (iv) to utilize the multicomponent reaction (MCR) as a vehicle for rapid SAR studies.

Regarding the development of target-directed inhibitors and single agents that provide target-directed inhibition of multiple disease-causing organisms or cells, we, amongst other things, have focused on trypanothione reductase (TryR), old aminoquinoline/aminoacridine drugs, and cysteine proteases (CPs). TryR has been identified in the causative agents of African trypanosomiasis (Trypanosoma brucei), Chagas disease (Trypanosoma cruzi), and leishmaniasis (Leishmania). It is a vulnerable genetically validated target for drugs that disrupt the natural redox defense system against oxidative stress in the aforementioned parasitic protozoa, the kinetoplastids [5-8]. This defense system is dependent upon trypanothione, $\mathrm{T}[\mathrm{SH}]_{2}$, which is also known as $N^{1}, N^{8}$-bis(glutathionyl)spermidine. $\mathrm{T}[\mathrm{SH}]_{2}$ is a low-molecular-weight dithiol that is absent from humans, but used by the kinetoplastids to maintain their intracellular redox balance. In the kinetoplastids, $\mathrm{T}[\mathrm{SH}]_{2}$ acts to counteract environmental stress through various enzymatic and nonenzymatic processes, and has been implicated in acquired resistance to chemotherapeutic agents [9]. In addition to the genetic validation of TryR, the discovery that the targets of several of the current antiprotozoal drugs are enzymes in the trypanothione metabolism further underscores the importance of TryR as an attractive target [10]. Despite the presence of the human homolog glutathione reductase (hGR), the differences in charge and size of the respective active sites of TryR and hGR have made it possible to design selective inhibitors of TryR. Indeed, a large number of TryR-specific inhibitors have already been identified [11,12]. Our work in this area is based upon the well-established old antimalarial drug quinacrine $\mathbf{1}$ and has led to new antiprotozoal inhibitors of TryR exemplified by urea 2, Fig. 1 [13].
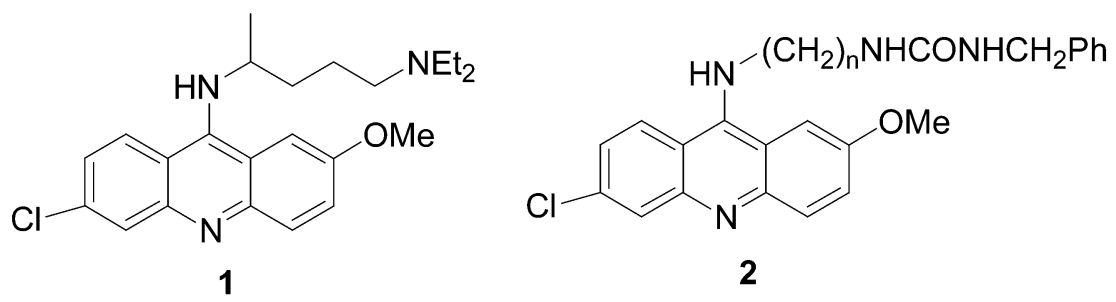

Fig. 1 Chemical structures of quinacrine and antiprotozoal urea derivatives. 
Chloroquine $\mathbf{3}$ is another old drug that has formed a basis for our medicinal chemistry program on single agents aimed at multiple targets within the malaria parasite Plasmodium falciparum. It is postulated that chloroquine and related quinoline antimalarials accumulate in the parasite's acidic food vacuole and inhibit $\beta$-haematin formation. Toxic haem thus builds up in the vacuole, subsequently killing the parasites [14]. Though the mode of action of these agents is still unclear, they remain potential sources of antimalarial therapy. This is partly because $P$. falciparum has had difficulty developing rapid resistance to this class of compounds. For example, simple modification of the lateral side chain of chloroquine has resulted in new derivatives, with activity against chloroquine-resistant strains $[14,15]$.

Our contribution to SAR studies of chloroquine-based antimalarials has focused on new metallocene analogs related to ferroquine $\mathbf{4 a}$, which is currently in clinical trials for the treatment of chloroquine-resistant malaria [16]. Our SAR studies have resulted in the identification of new ferroquine and related derivatives 4-7, Fig. 2 [17-19]. These studies were conducted in order to establish the role of the iron/ferrocene in ferroquine. The synthesis of the ruthenocene analogues $\mathbf{4 b}, \mathbf{5 b}, \mathbf{6 b}$, and $\mathbf{7 b}$ resulted in the unexpected synthesis of 4-aminoquinolines with 1,1'-disubstituted ruthenocenes in the side chain, e.g., (6b) (isoruthenoquine) and (7b). We have also had to develop new synthetic routes since ferrocene and ruthenocene differ significantly in reactivity $[18,19]$. More importantly, the aforementioned SAR studies have resulted in the discovery of phenylenequine $\mathbf{8}$, which is significantly more active in vitro than chloroquine against resistant strains. At present, phenylenequine is undergoing in vivo studies in mice and preliminary data suggest it is also active in vivo.

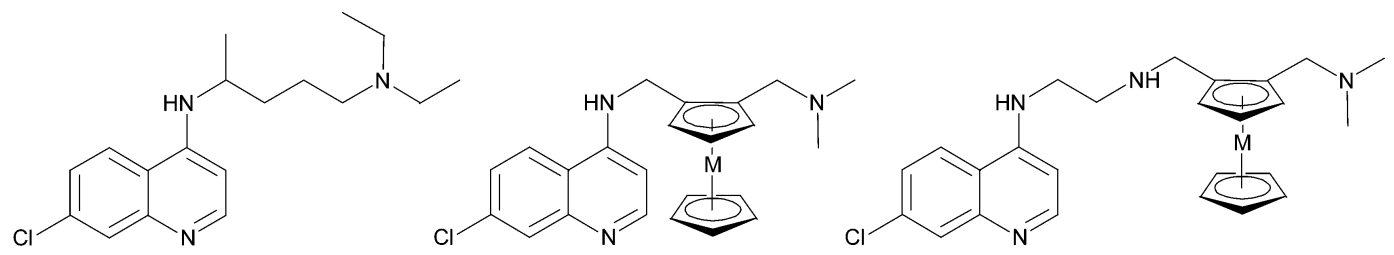

3<smiles>CN(C)Cc1ccccc1NCc1cccc(Nc2cccc3ncccc23)c1</smiles>

$6 \mathrm{a} M=\mathrm{Fe}$

$6 b M=R u$
$4 \mathrm{a} M=\mathrm{Fe}$

4b $M=R u$

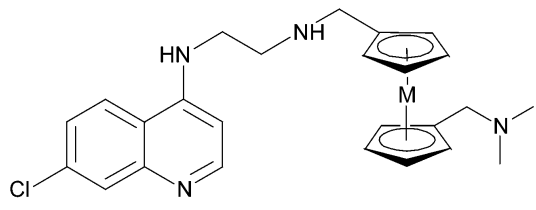

$7 \mathrm{a} M=\mathrm{Fe}$

$7 \mathrm{~b} \mathbf{M}=\mathbf{R u}$
$5 \mathrm{a} M=\mathrm{Fe}$

5b M $=\mathbf{R u}$<smiles>CN(C)Cc1ccccc1CNc1ccnc2cc(Cl)ccc12</smiles>

8

Fig. 2 Chemical structures of chloroquine and new analogs.

On the other hand, CPs in protozoan parasites are attractive targets for the development of antiparasitic chemotherapy due to the key role they play at various stages in the life cycle of the parasites. For example, one recent promising approach to new antimalarial agents has been the use of proteases as biological targets. Proteases play crucial roles in the metabolism, replication, survival, and pathology of parasites in general. Within the context of malaria, among potential new targets for antimalarial chemotherapy are enzymes involved in hemoglobin degradation. Intraerthrocytic Plasmodium trophozoites derive amino acids for protein synthesis from the hydrolysis of host cell hemoglobin. This occurs in the food vacuole, and the proteases involved include members of the aspartic protease [20,21], cysteine protease [22-25], and metalloprotease [26] families. Thus, development of CP inhibitors that would interfere with the malaria parasite's ability to degrade and utilize hemoglobin as a source of nutrients has become a viable strategy in the search for new antimalarial drugs [27]. These inhibitors 
clearly have a novel mode of action, which is in marked contrast to the mode of action of existing clinically used antimalarial agents. Thus, cross-resistance is avoided. Indeed, CP inhibitors have been shown to be active in vitro against both the chloroquine-sensitive and -resistant strains of $P$. falciparum and in vivo to cure mice infected with the malaria parasite [28].<smiles>[R]c1cccc(COC(=O)n2c3c([R])cc([R])cc3c(=O)c(=O)n2[R]N2C(=O)C(=O)c3cc([R])cc([R2])c32)c1</smiles>

Fig. 3 Chemical structures of isatin parasitic CP inhibitors.

In the course of our research on antimalarial natural products [29], we have been exploring the potential of isatin 9 as a natural product antiprotozoal CP inhibitor scaffold [30]. Accordingly, exploratory compounds 10-12 were initially targeted (Fig. 3). We reasoned that the presence of the ketone functionality adjacent to the aromatic ring on the isatin scaffold provides a diversity point for accessing the corresponding thiosemicarbazones, a class of molecules that have been clinically evaluated over the last 50 years in a number of therapeutic areas. Of relevance to economic and target-based drug discovery for tropical diseases is the recent finding that simple aromatic thiosemicarbazones are antiprozoal CP inhibitors [31,32]. Coupled with the molecular simplicity, potentially cost-effective synthesis and nonpeptidic nature, this makes isatins attractive natural product-based scaffolds for antimalarial drug discovery. Cost-effective synthesis should always be a consideration in deciding on potential new antiprotozoal agents given the prevalence of the major parasitic diseases in poor countries.

The simplicity of the chemistry involved in the synthesis of our isatin derivatives cannot be overemphasized. Compounds in the series 10-12 were prepared as depicted in Scheme 1. For the synthesis of 10, a series of commercially available isatin (1.0 equiv) were treated with benzyl halides (1.2 equiv) in $\mathrm{CH}_{2} \mathrm{Cl}_{2}$ as solvent in the presence of 4.0 equiv of potassium fluoride on alumina $\left(\mathrm{KF} / \mathrm{Al}_{2} \mathrm{O}_{3}\right)$. The reaction mixtures were stirred at room temperature for $16 \mathrm{~h}$, after which the resultant mixtures were simply diluted with diethyl ether and the crude isolated. Purification of the crude reaction mixtures by column chromatography over silica gel (3:7; EtOAc:hexane) generally gave high yields of the desired products. During our method development, we observed that 4 equiv of the base were necessary to drive reactions to completion.

The synthesis of derivatives 11 was achieved by reaction of isatin/5-substituted isatins (1.0 equiv), dissolved, and stirred in $\mathrm{CH}_{3} \mathrm{CN}$ at $0{ }^{\circ} \mathrm{C}$ in an ice bath, with benzyl chloroformate (1.1 equiv) in the presence of 0.1 equiv of 4-dimethylaminopyridine (DMAP) and triethylamine (1.1 equiv). After stirring for $3 \mathrm{~h}$ at $0{ }^{\circ} \mathrm{C}$, the desired products were obtained by filtration from the reaction mixture, washed with cold ethanol, and dried to give varying yields of the products. Thiosemicarbazones 12 were prepared by simple condensation of the commercial isatins and thiosemicarbazide. 
12 $40-98 \%$

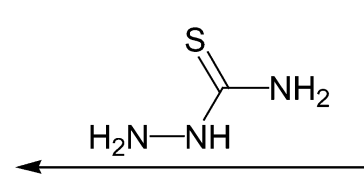

$\mathrm{EtOH}, 45{ }^{\circ} \mathrm{C}$<smiles>[R]c1cc([R2])c2c(c1)C(=O)C(=O)N2</smiles><smiles>[Na][Mg][Mg]</smiles>

10

$\mathrm{R}^{3}-\mathrm{X}$, solvent $48-94 \%$<smiles>CCN(C)CCN(C)C(=O)OCc1ccccc1</smiles>

Scheme 1 Synthesis of compounds 10-12.

In terms of the biological results, synthesized compounds were initially tested against recombinant falcipain-2. Commercially available isatins were screened for comparison purposes and were found to be ineffective with the exception of 5-nitro isatin, which showed an $\mathrm{IC}_{50}$ value of $43 \mu \mathrm{M}$. On the other hand, thiosemicarbazones showed some activity (albeit modest in many cases). The most notable thiosemicarbazone scaffold from this limited series are 12g and 12I, which showed activity against the target protease with $\mathrm{IC}_{50}$ values of $10 \mu \mathrm{M}$ or less (Fig. 4).<smiles>[R]c1cc([N+](=O)[O-])cc2c1NC(=O)/C2=N/NC(N)=S</smiles>

Fig. 4 Chemical structures of isatin parasitic CP inhibitors.

The most notable compound from the ketone series of $N$-substituted derivatives is $\mathbf{1 0 h}$, which showed activity against falcipain- 2 with an $\mathrm{IC}_{50}$ value of $9.2 \mu \mathrm{M}$. The potency against falcipain- 2 of compounds arising from $\mathrm{N}$-benzylation may be attributed to the ability of the benzyl moiety to bind more tightly in the $S_{2}$ pocket of the active site of the enzyme [30]. Proteases bind peptide substrates in their active site cleft through backbone and side chain interactions that lie around several defined pockets in the enzyme termed S1, S2, S3, S4 for those pockets that bind to the corresponding amino acids that are N-terminal to the scissile amide bond, while the S1', S2', S3', S4' pockets bind to the amino acids C-terminal to the scissile bond. On the other hand, the increased activity $\left(\mathrm{IC}_{50}=4.4 \mu \mathrm{M}\right)$ of thiosemicarbazone 12g may be due to the electron-withdrawing nitro group, which renders this compound more susceptible to nucleophilic attack by the active site cysteine thiol. This nucleophilic attack could occur either at the imine carbon as with peptidyl semicarbazones (Fig. 5) or the thiocarbonyl group as recently suggested for cruzain, a related parasitic CP from T. cruzi (Fig. 6) [31]. 


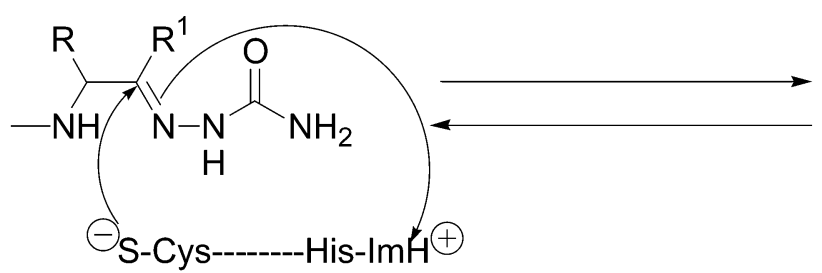<smiles>[R]C(NC)C([R])(NNC(N)=O)S[GeH3]</smiles>

Fig. 5 Inhibition of $\mathrm{CP}$ by peptidyl semicarbazones.

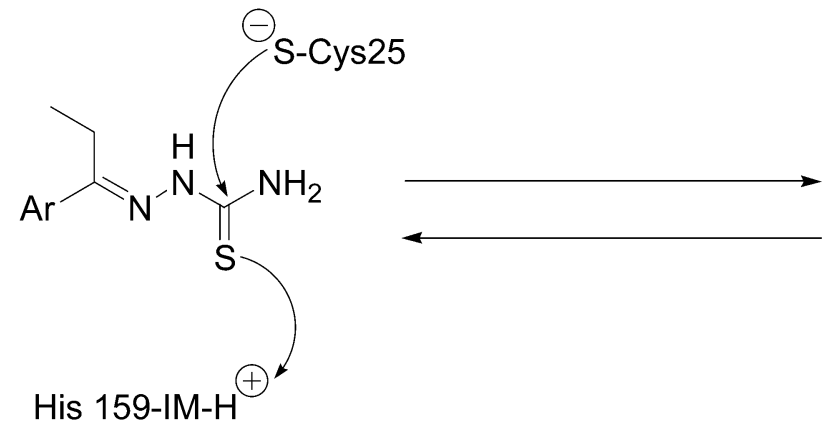<smiles>CC/C([Al])=N\NC(N)(S)S[Ge]</smiles>

His 159-Im

Fig. 6 Inhibition of $\mathrm{CP}$ by thiosemicarbazones.

Our work on thiosemicarbazone parasitic CP inhibitors suggests that the mechanism of action of thiosemicarbazones is complex and may be mediated through inhibition of multiple targets including CPs [32]. We have synthesized a library of tridentate chelating thiosemicarbazones $\mathbf{1 3}$ (Fig. 4) and screened them against three parasitic CPs, cruzain, falcipain-2, and rhodesain and against the respective parasite sources of these three proteases, T. cruzi, P. falciparum, and T. brucei. The screens identified compounds that were effective against the enzymes and the parasites, but also some compounds that were parasiticidal despite a lack of activity against the proteases [32].

The advent of combinatorial chemistry has in the recent past emerged as a powerful tool for the rapid generation, identification, and optimization of lead compounds in the drug discovery process. MCRs, reactions in which more than two starting materials all present together in one reaction vessel combine to form a final product, have in this regard been used to efficiently generate chemical diversity in the final products in a few reaction steps. Compared with conventional organic reactions, MCRs are advantageous in being highly convergent and in requiring minimum effort to achieve. Several MCRs are known to date, among which the Ugi 4 component condensation (U-4CC) of amine, oxo compound, carboxylic acid, and isocyanide, and its variants remains, by far, the most documented and most versatile. Coupled with combinatorial chemistry, MCRs have been used to generate chemical diversity in a few reaction steps and usually in a one-pot operation. Our interest in applying MCRs to antimalarial drug discovery stems from this attractive feature and the realization that the rational choice of inputs based on known antimalarial bioactiphores or pharmacophores could lead to the generation of diverse biologically rich libraries designed to explore rapid SARs.

We have so far applied the U-4CC to generate new aminoquinolines 14 [33]. Synthesis of the target compounds was achieved in methanol at room temperature in parallel array format in yields ranging from $60-74 \%$ (Scheme 2). Antimalarial screening of the derivatives revealed a potent compound with an $\mathrm{IC}_{50}$ value of $73 \mathrm{nM}$ against a chloroquine-resistant $\mathrm{K} 1$ strain in which the $\mathrm{IC}_{50}$ value of chloroquine is around $300 \mathrm{nM}$. 
<smiles>Nc1cnc2cc(Cl)ccc2c1</smiles><smiles>O=C(O)c1ccccc1</smiles>

$\mathrm{CH}_{2} \mathrm{O}$

$\mathrm{R}^{1} \mathrm{NC}$

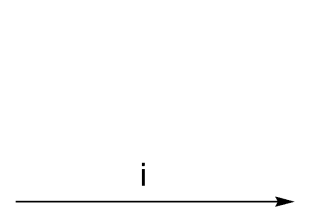<smiles>[R]NC(=O)CN(CCNc1ccnc2cc(Cl)ccc12)C(=O)c1ccccc1[R]</smiles>

Scheme 2 Synthesis of target compounds. Reagents and conditions: (i) MeOH, rt, 36-48 h.

Currently, we are designing MCR inputs in such a way that the final product is aimed at multiple targets within the malaria parasite and other disease-causing organisms. This approach could be useful in potentially slowing down or even overcoming the emergence of drug resistance and is analogous to conventional combination therapy wherein two or more antimalarial drugs, with different mechanisms of action, are coadministered.

\section{CONCLUSION}

It is very clear that the scaling down and/or de-emphasizing by many pharmaceutical companies of natural products as sources of novel drug leads in preference to classical combinatorial libraries has had detrimental effects on productivity in drug discovery. While African scientists do not have the luxury of access to large synthetic chemical libraries, they have a powerful resource in natural products that are uniquely endemic to the continent. The challenge for the African scientist is to depart from the traditional culture of isolation-structural elucidation of plant and/or animal products lacking meaningful bioassay, chemosystematics, taxonomy, and medicinal chemistry. There must be a total paradigm shift to a truly multidisciplinary approach in African natural products research. In fact, one of the immediate requirements of the African natural products chemistry community is to realize the potential for collaboration in lead optimization through medicinal chemistry and synthetic organic chemistry with attendant interfaces with biological function and processes. Such a paradigm shift is likely to contribute immensely to the training of students and future scientists, capable of competing internationally, in multidisciplinary research.

\section{ACKNOWLEDGMENTS}

I would like to thank my numerous scientific collaborators, but especially Profs. Philip J. Rosenthal and James H. McKerrow (University of California, San Francisco, USA), Dr. Doron Greenbaum (Walter and Eliza Hall Institute of Medical Research, Australia), Prof. Peter J. Smith (University of Cape Town), and Prof. Alan H. Fairlamb (Wellcome Trust Biocentre, University of Dundee, UK). I would also like to thank the following for financial support of my research programs over the years: National Research Foundation of South Africa, the South African Department of Trade and Industry through the THRIP program, GlaxoSmithKline (UK), The Wellcome Trust (UK), NIH (USA), Merck Research Laboratories (USA), the South African Medical Research Council, the South African Innovation Fund Trust, Cancer Association of South Africa, and the University of Cape Town. 


\section{REFERENCES}

1. D. J. Newman, G. M. Cragg, K. M. Snader. J. Nat. Prod. 66, 1022 (2003).

2. T. N. Raju. Lancet 355 (2000).

3. J. R. Proudfoot. Bioorg. Med. Chem. Lett. 12, 1647 (2002).

4. C. A. Lipinski, F. Lombardo, B. W. Dominy, P. J. Feeney. Adv. Drug Delivery Rev. 23, 3 (1997).

5. A. H. Fairlamb, P. Blackburn, P. Ulrich, B. T. Chait, A. Cerami. Science 227, 1485 (1985).

6. A. H. Fairlamb and A. Cerami. Annu. Rev. Microbiol. 46, 695 (1992).

7. S. Muller, E. Liebau, R. D. Walter, R. L. Krauth-Siegel. Trends Parasitol. 19, 320 (2003).

8. C. Dumas, M. Ouellette, J. Tovar, M. L. Cunningham, A. H. Fairlamb, S. Tamar, M. Olivier, B. Papadopoulou. EMBO J. 16, 2590 (1997).

9. A. Schmidt and R. L. Krauth-Siegel. Curr. Top. Med. Chem. 2, 1239 (2002).

10. D. S. Fries and A. H. Fairlamb. In Burger's Medicinal Chemistry and Drug Discovery: Chemotherapeutic Agents, D. J. Abraham (Ed.), pp. 1033-1087, John Wiley, New York (2003).

11. K. Chibale and C. C. Musonda. Curr. Med. Chem. 10, 1863 (2003).

12. K. Augustyns, K. Amssoms, A. Yamani, P. K. Rajan, A. Haemers. Curr. Pharm. Des. 7, 1117 (2001).

13. K. Chibale, H. Haupt, H. Kendrick, V. Yardley, A. Saravanamuthu, A. H. Fairlamb, S. L. Croft. Bioorg. Med. Chem. Lett. 11, 2655 (2003).

14. T. J. Egan. Drug Design Rev.—Online 1, 93 (2004).

15. D. De, F. M. Krogstad, L. D. Byers, D. J. Krogstad. J. Med. Chem. 42, 4918 (1998).

16. C. Biot. Curr. Med. Chem., Anti-Infective Agents 3, 135 (2004).

17. K. Chibale, J. R. Moss, M. Blackie, D. van Schalkwyk, P. J. Smith. Tetrahedron Lett. 41, 6231 (2000).

18. P. Beagley, M. A. L. Blackie, K. Chibale, C. Clarkson, J. R. Moss, P. J. Smith. J. Chem. Soc., Dalton. Trans. 4426 (2002).

19. P. Beagley, M. A. L. Blackie, K. Chibale, C. Clarkson, R. Meijboom, J. R. Moss, P. J. Smith, H. Su. Dalton Trans. 3046 (2003).

20. S. E. Francis, D. J. Sullivan, Jr., D. E. Goldberg. Annu. Rev. Microbiol. 51, 97 (1997).

21. R. Banerjee, J. Liu, W. Beatty, L. Pelosof, M. Klemba, D. E. Goldberg. Proc. Natl. Acad. Sci. USA 99, 990 (2002).

22. P. J. Rosenthal. Curr. Opin. Hematol. 9, 140 (2002).

23. B. R. Shenai, P. S. Sijwali, A. Singh, P. J. Rosenthal. J. Biol. Chem. 275, 29000 (2000).

24. P. S. Sijwali, B. R. Shenai, J. Gut, A. Singh, P. J. Rosenthal. Biochem. J. 360, 481 (2001).

25. P. S. Sijwali, K. Kato, K. B. Seydel, J. Gut, J. Lehman, M. Klemba, D. E. Goldberg, L. H. Miller, P. J. Rosenthal. Proc. Natl. Acad. Sci. USA 101, 8721 (2004).

26. K. K. Eggleston, K. L. Duffin, D. E. Goldberg. J. Biol. Chem. 274, 32411 (1999).

27. P. J. Rosenthal, P. S. Sijwali, A. Singh, B. R. Shenai. Curr. Pharm. Des. 8, 99 (2002).

28. J. E. Olson, G. K. Lee, A. Semenov, P. J. Rosenthal. Bioorg. Med. Chem. 7, 633 (1999).

29. C. Clarkson, C. C. Musonda, K. Chibale, W. E. Campbell, P. J. Smith. Bioorg. Med. Chem. 11, 4417 (2003).

30. I. Chiyanzu, E. Hansell, J. Gut, P. J. Rosenthal, J. H. McKerrow, K. Chibale. Bioorg. Med. Chem. Lett. 13, 3527 (2003).

31. X. Du, C. Guo, E. Hansell, P. S. Doyle, C. R. Caffrey, T. P. Holler, J. H. McKerrow, F. E. Cohen. J. Med. Chem. 45, 2695 (2002).

32. D. Greenbaum, Z. Mackey, E. Hansell, P. Doyle, J. Gut, C. R. Caffrey, J. Lehman, P. J. Rosenthal, J. H. McKerrow, K. Chibale. J. Med. Chem. 47, 3212 (2004).

33. C. C. Musonda, D. Taylor, J. Lehman, J. Gut, P. J. Rosenthal, K. Chibale. Bioorg. Med. Chem. Lett. 14, 3901 (2004). 